\title{
Molecular and Microbial Studies Survey for Camel Udder Microbiota
}

\author{
Zayed Alnefaie1, Mohamed Abu-Zeid ${ }^{1,2}$, Mohammed H. Mutwakil1, Mohamed Morsi M. Ahmed1,3 \\ ${ }^{1}$ Department of Biological Sciences, Faculty of Science, P.O. Box 80203, King Abdelaziz University, Jeddah, 21589, Saudi Arabia. \\ ${ }^{2}$ Microbial Genetics Department, Genetic Engineering and Biotechnology Division, National Research Center, Dokki, Giza, Egypt. \\ ${ }^{3}$ Nucleic Acids Research Dept., Genetic Engineering and Biotechnology Research Institute (GEBRI), City for Scientific Research and Technological Applications. \\ Alexandria, Egypt.
}

Correspondence Author: Zayed Alnefaie, Department of Biological Sciences, Faculty of Science, P.0. Box 80203, King Abdelaziz University, Jeddah, 21589, Saudi Arabia.

E-mail: zayedalnefaie@gmail.com

Received date: 12 November 2018, Accepted date: 10 February 2019, Online date: 25 February 2019

Copyright: (C) 2019 Zayed Alnefaie et al., This is an open-access article distributed under the terms of the Creative Commons Attribution License, which permits unrestricted use, distribution, and reproduction in any medium, provided the original author and source are credited.

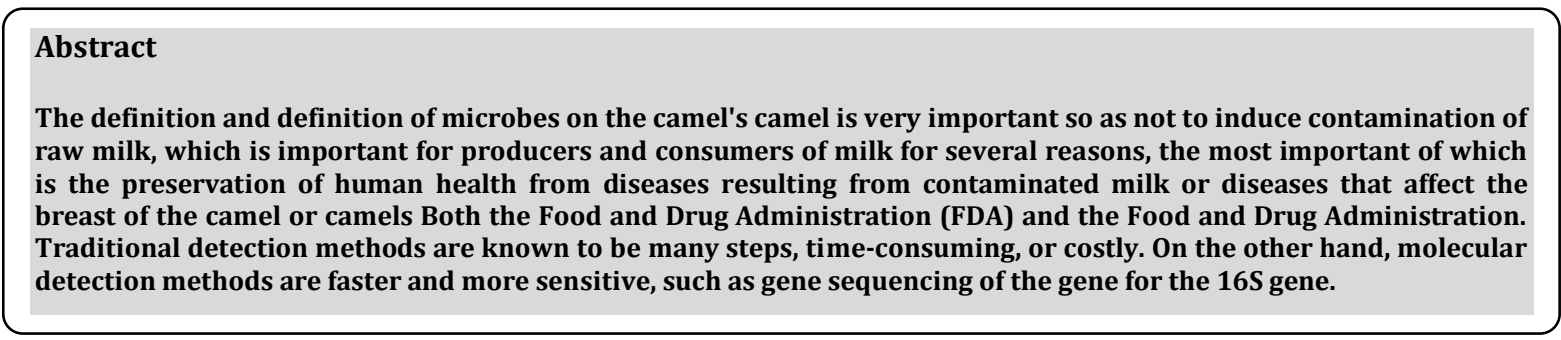

Key words: Molecular detection, PCR, camel, udder, microbiota, 16s rRNA gene

\section{INTRODUCTION}

The camel is well adapted to harsh climatic conditions and due to this it forms a sustainable livelihood for the pastoral communities by providing the following: Production of meat, milk, labor, means of transport and as a genetic resource base which is much available that can be exploited for livelihoods of pastoral communities [1]. The current rapid human population growth, increased levels of urbanization, coupled with the current low economic status of the counties have negatively impacted on the camel. Camels were best adapted to the harsh environments and fluctuating nutritional conditions of the arid and extremely arid zones [2]. The significant challenges in pastoral livestock production including camels are feeding, health and housing.

The increased incidence of diseases is one of the limitations associated with camel production [3]. Gastrointestinal parasites like Trematodes, Cestodes, and Nematodes are known to contribute to loss of reproductive and productive performance in camels [4]. The primary clinical signs of parasitic gastroenteritis include severe diarrhea, stomach pain, and weight loss, reduce production rate, decreased feed intake, and increased veterinary cost and subsequent death in severe cases. Animals in pain and discomfort are less productive than their healthy counterpart. Some parasites have zoonotic implications to those who work closely with camels [5]. Studies have shown that climate, management system, inadequate husbandry and underfeeding have an influence on occurrence and pattern of infection among camel populations. Presence of various helminths species with high prevalence is an indication of favorable environmental condition for infection, survival, and perpetuation of parasite existence in camel [3].

With the Advent of whole-genome sequencing, it is by and by possible to unequivocally assemble how bacterial universe can disseminate and conform to different headways are scale. Current genomic approaches are nisus to discover the premise of various bacterial phenotypes by predicting the result of flexible changes occurring in the midst of the improvement of mortal masses. Likewise, genomic data can be used to make laying claim about the natural conditions and transformative climatic insistency experienced in another bacterial storehouse, and on the microbial bionomics and not too bad assortment of different organic groups.

The constant adjustment of human pathogens is encapsulated by the development and multiplication of anti-toxin safe microscopic organisms in present-day progress. In this manner, translating how these genomic changes happen has substantial ramifications at a central level, in better understanding the science of bacterial pathogens, yet additionally for the study of disease transmission and clinical purposes in deciding the best control and therapeutic techniques. The sharp pathogen Streptococcus 
agalactiae causes ailment in different hosts, most outstandingly in people and steers. In any case, its developmental history and host specificities are not by any stretch of the imagination comprehended. To address this, current genomic techniques give an inside and out approach of concentrate the host-organism collaboration of GBS so as to: find the most critical particular requirements; evaluate how GBS connects with the encompassing microbial groups and distinguish the qualities that are most regularly traded; decide the danger of between have transmission of GBS and help control its progressing dispersal. The present work survey to detect and identify microbes in camel udder microbiota.

\section{The host generalist pathogen Streptococcus agalactiae}

Streptococcus is a creature assortment that contains more than fifty saw sorts of $\mathrm{g}$ - a positive and balanced bacterium that can colonize an alternate scope of beast swarm [6]. While looking at infections in bovines, Nocard and Mollereau named a particular mastitis-causing Streptococcus, which they depicted Streptococcus no cardi [7]. A while later, this animal category was renamed Streptococcus agalactiae, intimating its negative impact on dairy animals like. Streptococci were parceled into two manufactured assembling: $\beta$-hemolytic and nonhemolytic, in light of their experience to cause the lysis of red parentage cells. A short time later, in 1933, Rebecca Lancefield also isolated $\beta$-hemolytic streptococci into a couple of class, according to a saccharide arranged on the cell surface of the minuscule life forms [8]. In this manner, S. agalactiae is comparably conveyed as the social event B Streptococcus (GBS) as it is the essential streptococcal species communicating the B antigen. GBS is a facultative anaerobe that can proficiently be refined in the Sir Alexander Robertus Todd Hewitt (TH) or mentality heart imbuement (BHI) medium.

Like several streptococci, it mannikin round chains that can be observed under a microscope, with the diameter of each cubicle ranging between 0 . sextet -ace $.2 \mathrm{um}$. In nature, these bacteria have a broad animal range but is most notably known for causing severe diseases in people, bovines, and marine animals. GBS inhabited the abdominal and genitourinary region of 10-30\% of a human being but appeared in the 1960s as a leading source of neonatal infections. In the adult population, colonization is mostly asymptomatic, although opportunistic infections can also occur among the elderly or in immune compromised individuals. In dairy camel, GBS clings to the mammary epithelium causing cow-like mastitis [9] while it was additionally observed to be in charge of flare-ups of intrusive ailment. Every single one of these repositories speaks to unmistakable biological specialties, mirroring the versatile capability of GBS to remarkably different host conditions.

\section{Bovine mastitis}

At first, in 1887, it is recognized GBS as a reason for ox-like mastitis [7]. Mastitis alludes to the provocative disease in the mammary organ, which can be divided into clinical or subclinical mastitis. The standard, favorite reason for aggravation is the nearness of pathogenic microorganisms in the udder. Despite the event that the teat skin cells can for the most part shield the udder from these irresistible operators after the sphincter muscles remain expanded for 1-2 hours, making the mammary organ be at an expanded the danger of contamination.

Amid this period, pathogenic living beings may trespass the nipple channel, duplicating and discharging poisons, catalysts and surface proteins. The level of the resistant reaction relies upon the lactation arrange, causative operator, age and wellbeing status [10,11]. For the most part, a substantial cell checks over 200000 cells/ml is demonstrative of mastitis [12,13]. Because of this physical cell increment, reducing its monetary esteem. In this way, mastitis is viewed as one of the costliest ailments of the dairy business, with a money-related weight extending all things considered from $61 €$ to $97 €$ for each tainted bovine every year, overall $[14,15,16]$. The appearance of more delicate culture-autonomous strategies has uncovered that the microbiota Colonization of Pregnant Female parent Rise Placental and Uterine Infection Pneumonia and Lung Injury Bacteraemia and Neonatal Infection comprises in a sophisticated group with numerous exceptional organic parts. Mastitis-causing operators can either be infectious, on the off chance that they are discovered exclusively in the udder or ecological if they additionally colonize the encompassing condition.

Frequently, dangerous life forms are in charge of more constant subclinical diseases, since they may go unnoticed for broadened timeframes. GBS is a standout amongst the common widely recognized infectious pathogens, close by different species, for example, S. aureus and Streptococcus dysgalactiae. Conversely, ecological operators are all the more effectively identified. GBS specifically, once thought as an entirely infectious pathogen transmitted, was as of late guaranteed to have an environmental course of transmission potentially. An investigation of Norwegian dairy ranches demonstrated that GBS could momentarily colonize the intestinal tract of steers, notwithstanding the drinking water and the horse shelter condition [16].

In any case, given that in each homestead examined in this work the GBS populace found in the earth is very homogeneous and like the one segregated from polluted steers, the nearness of GBS in the encompassing condition could be characteristic of crosssullying from infected bovines instead of genuine ecological colonization. Until the second 50\% of the twentieth century, GBS prevailed as a reason for intramammary contaminations, and soon after that a few control measures were set up to all the more proficiently treat tainted cows and to anticipate promote scattering inside a group [17.18]. Therefore, a considerable diminishment in the quantity of GBS contaminations was enrolled all through Europe. The predominance of GBS mastitis stays raised in singular nations, such as Germany while in others it has re-emerged.

\section{Colonization and virulence factors}

Numerous qualities and their relating items have been ensnared in the ability of GBS to colonize and contaminate its hosts. In any case, practical investigations have mostly centered around their part with regards to human contamination. Through mark labeled transposon mutagenesis (STM), a few genomic loci in charge of an assortment of bacterial procedures were at first appeared to be needed in a neonatal rodent sepsis contamination display. From that point forward, a few investigations have shown the part of 
particular variables for GBS contamination and colonization. The pathogenesis of GBS depends mainly on three systems: (I) capability to colonize and cross tissue hindrances inside the host condition; (ii) ability to sidestep the host protection components; and (iii) articulation of destructiveness parts that reason harm to the host.

\section{Surface polysaccharides}

Fundamental to all GBS strains is the gathering B-particular antigen (GBC), at first distinguished by Rebecca Lancefield. This peptidoglycan-tied down antigen is spoken to by an intricate structure made out of rhamnose, galactose, $\mathrm{N}$-acetylglucosamine, and glucitol. Despite the fact that the natural part of GBC is not altogether known, a current report demonstrated that GBC-insufficient mutant cells showed a few variations from the norm identified with morphology and evolution, proposing that GBC may comprise in an auxiliary segment of the cell divider. Surface antigens are critical supporters of the pathogenic capability of GBS, and a standout amongst the most crucial ones is the case polysaccharide (CPS). Anticapsular antibodies were appeared to give defensive invulnerability in a creature demonstrate, so particular intrigue has been set on the investigation of the container and its potential as an objective for immunization against GBS [19].

The case operon involves four preserved qualities having a place with the neu operon (neuA, neuB, neuC and neuD), six classes moderated over every single capsular compose and an extra factor set of six that decide the capsular kind of each strain (cpsF, cpsG, cpsH, cpsI, cpsJ, cpsK, cpsM). The neu qualities are in charge of the union of sialic corrosive and ensuing sialylation of the case. This procedure gives a vital capacity to the polysaccharide container, as it enables the microscopic organisms to oppose opsonophagocytosis by staying away from the enactment of substitute pathway of supplement framework [20]. Despite its vital part, the container polysaccharide is not communicated by a noteworthy, yet the factor, an extent of GBS segregates. In the human-inferred populace, they, as a rule, represent 5-20\% of the isolates, while in bovines 30-77\% of GBS strains are noncapsulated [20]. As of late, the atomic reason for case misfortune has been additionally examined, recommending that particular changes overwhelmingly inside cpsE were in charge of inactivation of case union [21]. These raises worry for future execution of a hostile to CPS immunization that is as of now being worked on to avert GBS colonization.

\section{Surface and secreted proteins}

Cell grip assumes a fundamental part of the survival and aggression of the host condition, and there are various proteins situated on the surface of the microorganisms connected with this procedure. One of the significant gatherings of surface proteins with the cement properties is portrayed by the nearness of an LPXTG theme that elevates covalent connection to the phone divider peptidoglycan. The hypervirulent GBS adhesin (HvgA) is a case of an LPXTG protein working as a fundamental destructiveness factor in GBS. It incorporates two significant variations: hvgA and bibA. Both comprise in a variable center with 50-60\% arrangement distinguish, flanked by moderated 5' and 3' closes. Vitally, it was demonstrated that HvgA is overexpressed in vivo and efficiently holds fast to intestinal epithelial cells. Additionally, explores shown that HvgA adds to the translocation of the intestinal obstruction and the intersection of the blood-mind boundary. Inside and out, these perceptions showed that Hvg A is the essential harmfulness include in GBS, related with the beginning of meningitis and LOD [22,23]. Two sorts of pilus-like structures have been recognized in gram-positive species: one portrayed by short, thin bars, while another spoke to by a more extended and more adaptable pilus. These pilin-particular compounds advance pilus polymerization, while the housekeeping transpeptidase SrtA is in charge of end of pilus get together and its covalent connection to the cell divider peptidoglycan through the LPXTG theme. Every segment of the pilus locus was appeared to assume a fundamental part of GBS colonization and malady movement.

The auxiliary proteins help start adherence to the animal tissue, though the spine protein additionally adds to intrusion and translocation inside the host cells. In particular, in GBS, three unmistakable genomic islands have been discovered: Pilus is land 1 (PI-1), Pilus island 2a (PI-2a) and Pilus island 2b (PI-2b). For PI-2, the two genomic variations PI-2a and PI-2b are unrelated inside GBS, as they share a similar addition site. Notwithstanding, utilitarian contrasts between the two alternatives have been portrayed. Of the two PI-2 islands, PI-2a was mainly involved in biofilm development, while the Spb1 protein inside PI-2b was uncovered to add to an upgraded survival inside macrophages.

\section{General concepts of molecular evolution}

Development is characterized as a procedure of progress that happens from age to age. In every single living being, the dominant part of unconstrained DNA changes happens because of blunders presented amid replication, which can cause fluctuating degrees of phenotypic repercussions. The established model and meaning of quality in bacterial genomes, first derived from pioneer concentrates, for example, those from François Jacob and Jacques Monod, has been refined all through the most recent decades. Quality can be depicted as a DNA area that can influence phenotypic characteristics through an articulation of a practical protein item. The promoter grouping of a class is the place the RNA polymerase, with a related sigma factor, will tie to start translation. At that point, inside the mRNA transcript, the ribosomal restricting site, otherwise called the ShineDalgarno succession, is where the ribosome will tie for protein interpretation to start and is generally like the accord grouping of AGGAGG. In conclusion, the purported coding arrangement (CDS) envelops a method of nucleotide triplets (codons) inside the mRNA that will be converted into a protein, regularly beginning in microscopic organisms from the amino corrosive $\mathrm{N}$-formylmethionine (fMet) up to a rubbish codon that will stop the interpretation procedure. These central principles deciding the stream of genetic data in bacterial living beings are seen in the dominant part of cases. 
Be that as it may, noncanonical components of interpretation can likewise be discovered [24,25] uncovering, for instance, the appearance of leaderless records for which review happens instantly toward the beginning of translation. Moreover, thinks about on non-coding RNAs (ncRNAs) have shown the usual assumption that all genetic data with natural ramifications is communicated as proteins [26].

\section{Phylogenetic inference}

At the point when two living beings advance from a typical precursor, they veer and autonomously get numerous transformations. Hence, the hereditary separation of couple life forms mirrors the level of uniqueness between them. The connection between the diverse subjects would then be able to be additionally settled by building a phylogenetic tree. The hereditary separation, and consequently the adjustments in DNA grouping, can be displayed as an arbitrary occasion around a particular trade demonstrate. The most direct model, called the JC69, computes hereditary separations were expecting that balance repetitions of every nucleotide are $25 \%$ each and that any nucleotide replacement has a similar odd of happening. On the other outrageous is a general time reversible (GTR) show [27], Which evaluates the recurrence of every nucleotide base and the rate at which each conceivable nucleotide substitution happens, while accepting that symmetric changes occur at a similar rate. Over parameterization can be an issue when utilizing more mind-boggling models, as estimating more parameters independently will build the general level of change and vulnerability in the investigation. Hence, the general suggestion is to survey how well each model fits with the tried dataset utilizing strategies to locate the one that contains as much unpredictability as required for a dependable portrayal. Another factor to consider while evaluating the rate of nucleotide substitution is the means by which it changes between various positions inside the grouping. It is natural learning that transformative rate contrasts between every codon position, with a decent starting point advancing the slowest and the third one the speediest. This is a consequence of various transformative weights acting at every area, as the shot of adjusting the amino corrosive coded is the most elevated in the primary codon base. Hence, conting ent upon the dataset, a model that considers rate variety among destinations might be required to derive precise hereditary separations. In microscopic organisms, a noteworthy puzzling component of the transformative flag is homologous recombination. By supplanting the genomic piece of one cell by a homologous succession show in a more far off life form, noteworthy variety might be acquainted with a beneficiary bacterium. Level quality exchange (HGT) can likewise include the securing or loss of novel, non-homologous hereditary sections through versatile hereditary components (MGEs). These genomic districts cause more critical transformative changes by possibly conveying new capacities to the populace that may enable it to flourish in its condition. Exchange can happen through transformation, conjugation or transduction. Evaluating the transformative rate Early perceptions of the hereditary separation between comparable proteins proposed that variety increments directly with the difference time. This offered to ascend to the idea of an atomic clock, a steady transformative rate at which every quality amasses new changes after some time. Like this, by knowing the developmental rate for a given quality or animal groups, inductions on their uniqueness time can be made, and the other way around. With the dissimilarity rate of a bacterial populace, we would then be able to date past transmission occasions and gauge to what extent particular ancestries have been colonizing their condition. Notwithstanding, experimental investigations have demonstrated that numerous qualities develop at a variable rate, which exhibited new difficulties for sub-atomic dating [29]. Current phylogenetic techniques, for instance, those in the "Bayesian Evolutionary Analysis Sampling Trees" (BEAST) program oblige some clock designs to either assess one worldwide and consistent rate for the populace (severe clock model) or adopt a more flexible strategy [30]. The region atomic clock demonstrates, at first proposed by Yoder and Yang, enables the rate of increase to be consistent inside special collections of the phylogenetic tree while accepting the other rate for more remote branches. Along with similar lines, the autocorrelated free clock show [29]. Is fixated on the suspicion that the transformative rate is more comparable among as of late veered taxa, so more prominent variety lies between the old and terminal branches. In conclusion, the uncorrelated loose clock models accept a branch-particular rate with no connection with the developmental records of the populace [28].

\section{Detecting natural selection}

The nearness of healthy choice can be construed in some ways. Since changes are required to emerge arbitrarily over the genome, watching intermittent substitutions at particular positions or qualities all through the populace may propose the nearness of an equal developmental weight following up on autonomous genealogies. In any case, a higher than anticipated substitution rate can likewise be because of varieties in transformation rate between qualities, or undetected recombination. Along these lines, this comprehensive approach ought to be additionally supplemented by examination of the heading of collective choice. This is derived by computing the rate of collection of nonsynonymous changes per nonsynonymous site (dN) about the rate of synonymous substitutions per synonymous site $(\mathrm{dS})$. On a fundamental level, nonsynonymous changes have a significant effect on protein structure and capacity. Uncorrelated lognormal atomic clock Autocorrelated sub-atomic clock Local sub-atomic clock 50 the protein is higher $(\mathrm{dN} / \mathrm{dS}>1)$ than what might be normal under nonpartisan development, this is demonstrative of positive choice. By differentiate, a dN/dS $<1$ proposes the nearness of sanitizing determination and the protection of protein work. The bearing of common choice, gathered with $\mathrm{dN} / \mathrm{dS}$, can be estimated at singular locales, over the specific arrangement of qualities or over the whole genome. Practically speaking, there are a few strategies to acquire the dN/dS proportion. One of the least complicated models, the Nei-Gojobori, includes surveying the average number of nonsynonymous and synonymous codons inside particular locales or qualities and standardize the watched number by this desire. In microbial genomics, given that arrangement information is as a rule as the arrangement of variable positions, computation of $\mathrm{dN} / \mathrm{dS}$ can be gathered from demonstrating the 
standard nonsynonymous rate for every conceivable nucleotide substitution $[8,32]$. Despite the fact that the $\mathrm{dN} / \mathrm{dS}$ proportion can be a helpful metric to gather the nearness of usual determination, it ought to be deciphered with an alert. For instance, the modest number of changes happening per site may restrict the measurable hugeness of $\mathrm{dN} / \mathrm{dS}$. In this manner, to expand its real power, $\mathrm{dN} / \mathrm{dS}$ can be ascertained per quality or gatherings of varieties. Be that as it may, by averaging out the dN/dS over different locales, the nearness of few emphatically chose changes inside a quality might be veiled and thought little of by an excess of picked contrarily substitutions) [32].

As already said, substitutions inside non-coding or intergenic areas may likewise have a critical natural criticalness. To have the capacity to surmise the effect of genomic variations inside non-coding fragments, there are a few instruments at present accessible, for example, spiff, that foresee the in all probability impact of transformations inside a given reference genome. In any case, this requires very much curated and commented on databases containing point by point data on promoter districts; administrators, translation begin locales and eliminators.

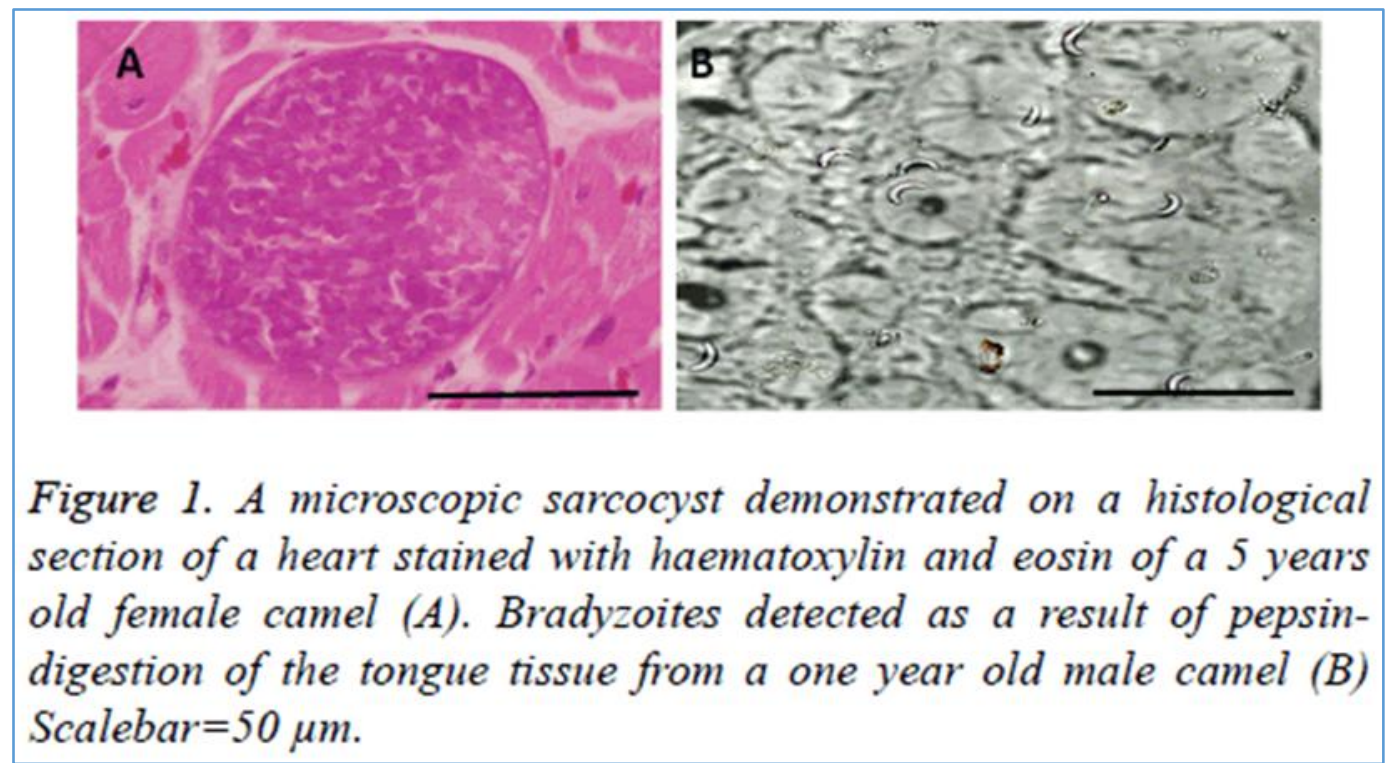

\section{CONFLICT OF INTEREST}

The authors declare no conflicts of interest

\section{ACKNOWLEDGMENT}

This work was supported by the King Abdulaziz City for Science and Technology in the Kingdom of Saudi Arabia 1-17010090012. The authors also, acknowledge assistance from the Science \& Technology Unit, Deanship of Scientific Research and Deanship of Graduate Studies, and acknowledge assistance from the Dept. of Biological Sciences, Faculty of Science, King Abdulaziz University (KAU), Jeddah, KSA.

\section{REFERENCES}

1. Schwartz H J, Dioli M. 1992. The One-Humped Camel (Camelus dromedaries) in Eastern Africa: A pictorial guide to diseases, health care, and management. Margraf Scientific Book Berlin, 283 pp.

2. Schwartz H J, Dolan Rosemary, Wilson AJ. 1983. Tropical Animal Health hand Production. 15: 169-178.

3. Swai E, Moshy W, Mshanga D, Lutatina J, Bwanga S. 2011. Intestinal parasitic infections of camels in the Agro and rural areas of Northern Tanzania. J. of Veterinary Research. 2: 34-38.

4. Schmindz GD. 1989. Foundations of Parasitology, 4th edition. Times minor/ College publishing, St. Louis. Tembely S, Diarra PA, Waigalo Y. 1992. Preliminary observations on helminth parasite populations of the dromedary in Northern Mali. Vet. Parasitol. 44(3-4), 339-342.

5. Mahmuda A, Mohammed A A, Alayande M, Habila Y, I Lawal M, Usman M, Raji A A, Saidu B, Yahaya M, Suleiman S. N.2014. Prevalence and distribution of GIT parasites of working camels in Sokoto metropolis.

6. Köhler W. 2007. The present state of species within the genera Streptococcus and Enterococcus. International Journal of Medical Microbiology.297 3:133-150.

7. Nocard N, Mollereau R. 1887. Sur une mammite contagieuse des vaches laitieres. Ann Inst Pasteur. 1: 109-26. 
8. Lieberman TD, et al. 2011. Parallel bacterial evolution within multiple patients identifies candidate pathogenicity genes. Nat. Genet. 43: 1275-1280.

9. Wyder A B, Boss R, Naskova, J, Kaufmann T, Steiner, A, Graber H U. 2011. Streptococcus spp. And related bacteria: their identification and their pathogenic potential for chronic mastitis - a molecular approach. Res. Vet. Sci. 91: 349-357.

10. Harmon RJ. 1994. Physiology of mastitis and factors affecting somatic cell counts. J. Dairy Sci. 77:2103.

11. Harmon RJ, Heald CW. 1982. Migration of polymorphonuclear leukocytes into the bovine mammary gland during experimentally induced Staphylococcus aureus mastitis. Am. J. Vet. Res. 43:992.

12. Ruegg PL. 2000. Milk quality premiums received by Wisconsin dairy farmers participating in directed milk quality programs. (abst)562 in Proceedings of the 9th Symp. Intl. Soc. Vet Epi and Econ. 6-11, Breckenridge, CO.

13. Ruegg PL, Tabone TJ. 2000. The relationship between antibiotic residues violations and somatic cell counts in Wisconsin dairy herds. J Dairy Sci. 83: 2805-2809.

14. Haghkhah M, Ahmadi MR, Gheisari HR, Kadivar A. 2011. Preliminary bacterial study on subclinical mastitis and teat condition in dairy herds around Shiraz. Turkish J. Vet. Anim. Sci. 35: 387-394.

15. Halasa T, Huijps K, østerås O, Hogeveen H. 2007. Economic effects of bovine mastitis and mastitis management: A review. Vet. Q. 29: 18-31.

16. Hogeveen H, Huijps K, lam T. 2011. Economic aspects of mastitis. New developments. New Zealand Vet. J. 59: 16-23.

17. Jorgensen HJ, Nordstoga AB, Sviland S, Zadoks RN, Solverod L, Kvitle B, Mork T. 2016. Streptococcus agalactiae in the environment of bovine dairy herds - Rewriting the textbooks? Vet Microbiol 184: 64-72.

18. Neave FK, Dodd FH, Kingwill RG, Westgarth DR.1969. Control of mastitis in the dairy herd by hygiene and management. J. Dairy. Sci 52 5:696-707.

19. Baker C, Rench M, Edwards M, Carpenter R, Hays B, Kasper D. 1988 Immunization of pregnant women with a polysaccharide vaccine of group B streptococcus. N Engl J Med 319: 1180-1185.

20. Marques M B, Kasper DL, Pangburn MK, Wessels MR. 1992. Prevention of C3 deposition by capsular polysaccharide is a viru- lence mechanism of type III group B streptococci. Infect. Immun. 60: 3986-3993.

21. Kong F, Lambertsen LM, Slotved Ko H-CD, Wang H, Gilbert GL. 2008. Use of phenotypic and molecular serotype identification methods to char- acterize previously nonserotypeable group B streptococci. J. Clin. Micro. biol. 46:2745-2750.

22. Rosini R, Rinaudo CD, Soriani M, Lauer P, Mora M, Maione D, et al. 2006. Identification of novel genomic islands coding for antigenic pilus-like structures in Streptococcus agalactiae. Mol Microbiol. 61:126-41.

23. Asmaa T, Disson O, Bellais S, Bouaboud A, Dmytruk N, Dramsi S, Mistou M, Khun H, Mechler C, Tardieux I, Trieu-Cuot P, Lecuit M, Poyart C. 2010. The surface protein HvgA mediates group B Streptococcus hypervirulence and meningeal tropism in neonates. J Exp Med 207: 2313-2322.

24. Tazi A, et al. 2010.The surface protein HvgA mediates group B Streptococcus hypervirulence and meningeal tropism in neonates. Journal of Experimental Medicine 207: 2313-2322.

25. De Groot A, Roche D, Fernandez B, Ludanyi M, Cruveiller S, Pignol D, et al. 2014. RNA Sequencing and Pro- to genomics Reveal the Importance of Leaderless mRNAs in the Radiation-Tolerant Bacterium Deino- coccus deserti. Genome Biol Evol. 6:932-48.

26. Vockenhuber MP, Sharma CM, Statt MG, Schmidt D, Xu Z, Dietrich S, et al. 2011. Deep sequencing-based identification of small non-coding RNAs in Streptomyces coelicolor. RNA Biol. 8:468-77.

27. Toledo-Arana A, Dussurget O, Nikitas G, Sesto N, Guet-Revillet H, Balestrino D, Loh E, Gripenland J, Tiensuu T, Vaitkevicius K, et al. 2009. The Listeria transcriptional landscape from saprophytism to virulence. Nature 459: 950-956.

28. Tavare' S. 1986. Some probabilistic and statistical problems in the analysis of dna sequences. Lectures on Mathematics in the Life Sciences American Mathematical Society 17: 57-86.

29. Jenkins GM, Rambaut A, Pybus OG, Holmes EC. 2002. Rates of molecular evolution in RNA viruses A quantitative phylogenetic analysis. J. Mol. Evol. 54: 156-165.

30. Drummond AJ, Suchard MA, Xie D, Rambaut A. 2012. Bayesian phylogenetics with BEAUti and the BEAST 1.7. Mol Biol Evol. 29:1969-1973.

31. Thorne JL, Kishino H, Painter IS. 1998. Estimating the rate of evolution of the rate of molecular evolution. Mol Biol Evol. 15:1647-1657.

32. Drummond AJ, Ho SYW, Phillips MJ, Rambaut A. 2006. Relaxed phylogenetics and dating with confidence. PLoS Biol. 4:699-710.

33. Lancefieldr C. 1933. Serological differentiation of human and other groups of hemolytic streptococci. J Exp Med. 57 4: $571-$ 595.

34. Hedge J, Wilson DJ. 2016. Practical Approaches for Detecting Selection in Microbial Genomes. PLoS Comput Biol 12(2): e1004739. 